\title{
Endothelial cation channel PIEZO1 controls blood pressure by mediating flow-induced ATP release
}

\author{
ShengPeng Wang, ${ }^{1}$ Ramesh Chennupati, ${ }^{1}$ Harmandeep Kaur, ${ }^{1}$ Andras Iring, ${ }^{1}$ Nina Wettschureck, ${ }^{1,2}$ and Stefan Offermanns ${ }^{1,2,3}$ \\ ${ }^{1}$ Max Planck Institute for Heart and Lung Research, Department of Pharmacology, Bad Nauheim, Germany. ${ }^{2}$ Centre for Molecular Medicine, University of Frankfurt, Frankfurt, Germany. ${ }^{3}$ Instituto de \\ Investigacion en Biomedicina de Buenos Aires (IBioBA), Buenos Aires, Argentina.
}

\begin{abstract}
Arterial blood pressure is controlled by vasodilatory factors such as nitric oxide (NO) that are released from the endothelium under the influence of fluid shear stress exerted by flowing blood. Flow-induced endothelial release of ATP and subsequent activation of $G_{q} / G_{11}$-coupled purinergic $P 2 Y_{2}$ receptors have been shown to mediate fluid shear stress-induced stimulation of NO formation. However, the mechanism by which fluid shear stress initiates these processes is unclear. Here, we have shown that the endothelial mechanosensitive cation channel PIEZO1 is required for flow-induced ATP release and subsequent $\mathrm{P} 2 \mathrm{Y}_{2} / \mathrm{C}_{\mathrm{q}} / \mathrm{C}_{11}$-mediated activation of downstream signaling that results in phosphorylation and activation of $A K T$ and endothelial NOS. We also demonstrated that PIEZO1-dependent ATP release is mediated in part by pannexin channels. The PIEZO1 activator Yoda1 mimicked the effect of fluid shear stress on endothelial cells and induced vasorelaxation in a PIEZO1dependent manner. Furthermore, mice with induced endothelium-specific PIEZO1 deficiency lost the ability to induce NO formation and vasodilation in response to flow and consequently developed hypertension. Together, our data demonstrate that PIEZO1 is required for the regulation of NO formation, vascular tone, and blood pressure.
\end{abstract}

\section{Introduction}

The ability of endothelial cells to respond to fluid shear stress exerted by blood flow is crucial for the development and function of the vascular system $(1,2)$. The relaxation of vascular smooth muscle in response to fluid shear stress sensed by the endothelium is a central autoregulatory function of the vessel, which adapts vessel diameter to blood flow and thereby controls vascular tone and blood pressure (3). Fluid shear stress triggers the release of various vasoactive mediators from endothelial cells, of which nitric oxide (NO), which is produced by the endothelial NOS (eNOS or NOS3), is regarded as the primary endothelial vasorelaxing factor (4-6). While the initial flow-sensing mechanism mediating flow-induced NO release and vasodilation is still rather unclear, previous work has identified several signaling processes, which link fluid shear stress to induction of NO formation (1, $2,7)$. An acute increase in flow leads to a transient intracellular $\left[\mathrm{Ca}^{2+}\right]$ $\left(\left[\mathrm{Ca}^{2+}\right]_{\mathrm{i}}\right)$ elevation, which then activates eNOS in a $\mathrm{Ca}^{2+} /$ calmodulindependent manner $(6,8,9)$. Sustained NO formation requires phosphorylation of eNOS by protein kinases such as AKT $(10,11)$ or protein kinase A $(6,12)$. Flow-induced activation of AKT involves a mechanosensory complex consisting of PECAM-1, VE-cadherin, and VEGFR2/VEGFR3 (13-16). Recent data indicate that flow-induced release of ATP from endothelial cells leads to the activation of the $\mathrm{G}_{\mathrm{q}} /$ $\mathrm{G}_{11}$-coupled purinergic $\mathrm{P} 2 \mathrm{Y}_{2}$ receptor on endothelial cells (17). This appears to be a critical upstream regulatory mechanism required for flow-dependent NO formation through the PECAM-1/VE-cadherin/ VEGFR mechanosensing complex and subsequent regulation of vascular tone in vitro and in vivo (17). However, the mechanism regulating flow-induced ATP release by endothelial cells is not known.

Conflict of interest: The authors have declared that no conflict of interest exists Submitted: February 29, 2016; Accepted: September 22, 2016.

Reference information: J Clin Invest. 2016;126(12):4527-4536. doi:10.1172/JCI87343.
The PIEZO proteins PIEZO1 and PIEZO2 are mechanically activated cation channels that form homomultimeric complexes $(18,19)$ sufficient to mediate mechanically induced currents $(18)$. PIEZO2 is the principal sensor of mechanical forces in mammalian Merkel cells (20-22), whereas PIEZO1 has been shown to mediate mechanically activated cation currents in various other cell types, including endothelial cells $(23,24)$. It has recently been shown that endothelial PIEZO1 can be activated by shear stress $(23,24)$ and that PIEZO1 is involved in shear stress-induced endothelial $\mathrm{Ca}^{2+}$ influx and cell alignment $(23,24)$. Consistent with a critical role of PIEZO1 in endothelial flow sensing in vivo, global and endothelial-specific loss of PIEZO1 results in severe defects in vascular development and remodeling, resulting in embryonic lethality $(23,24)$. While these data show that endothelial PIEZO1 can mediate endothelial flow sensing and is required for the development and maintenance of vascular architecture, the mechanisms downstream of endothelial PIEZO1 and its role in vascular tone and blood pressure regulation in adult mice remain unclear.

Here we demonstrate by in vitro studies and analysis of inducible, endothelium-specific PIEZO1 deficiency in adult mice that endothelial PIEZO1 mediates flow-induced ATP release and subsequent $\mathrm{P}_{2} \mathrm{Y}_{2} /$ $\mathrm{G}_{\mathrm{q}} / \mathrm{G}_{11}$-dependent activation of downstream signaling pathways, resulting in the regulation of vascular tone and blood pressure.

\section{Results}

PIEZO1 mediates endothelial responses to fluid shear stress in vitro. To analyze whether PIEZO1 is involved in endothelial mechanotransduction, we tested the effect of a siRNA-mediated knockdown of PIEZO1 on various shear stress-induced endothelial responses in human umbilical arterial endothelial cells (HUAECs). Knockdown of PIEZO1 strongly inhibited shear stress-induced increases in $\left[\mathrm{Ca}^{2+}\right]_{\mathrm{i}}$ (ref. 24 and Figure $1 \mathrm{~A}$ ) and also blocked phosphorylation of 
A

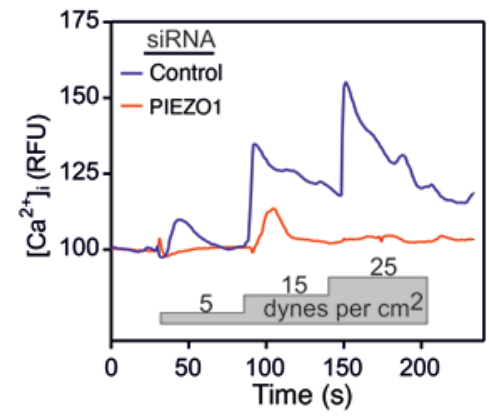

C

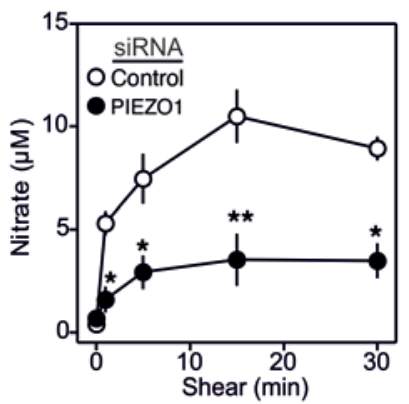

B
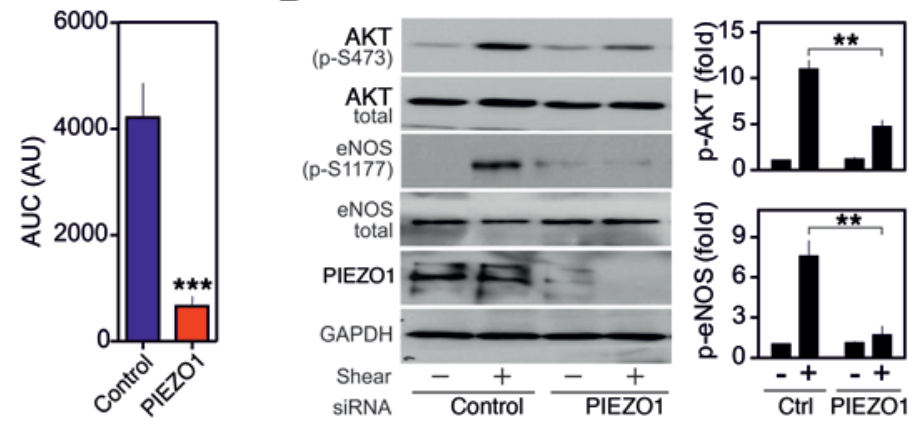

D
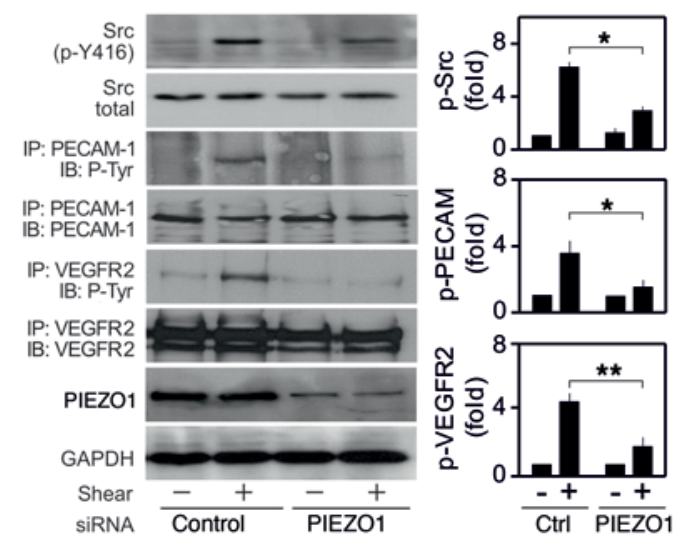

$\mathbf{E}$

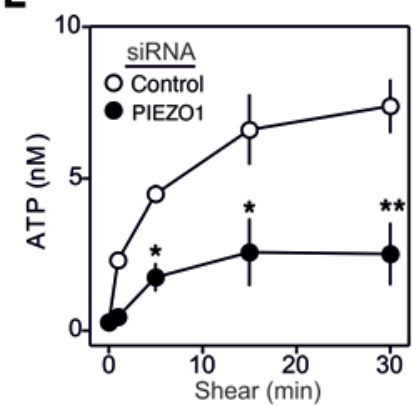

Figure 1. PIEZO1 mediates endothelial response to fluid shear stress in vitro. HUAECs were transfected with scrambled (control) siRNA or siRNA directed against PIEZO1 as described in Methods. (A) Fluo-4-loaded HUAECs ( $n=22$, control; $n=29$, PIEZO1 knockdown; 3 independent experiments) were exposed to the indicated shear forces, and $\left[\mathrm{Ca}^{2+}\right]$, was determined as fluorescence intensity (RFU, relative fluorescence units). Bar diagrams show areas under the curve (AUC). Shown are means \pm SEM; ${ }^{* * *} P \leq 0.001$ (2-tailed Student's $t$ test). (B-E) HUAECs ( $n=3$ ) were exposed to fluid shear (15 dyn/cm²) for 5 minutes in $\mathbf{B}$ and $\mathbf{D}$ or for the indicated time periods. For determination of Src activation, 15 seconds of shear was applied. AKT, eNOS, and Src activation (B and D) was determined by Western blotting for phosphorylated AKT, eNOS, and Src kinases and total AKT, eNOS, and Src. (C) Nitrate concentration in the cell medium. PECAM-1 and VEGFR2 activation (D) was determined by immunoprecipitation and Western blotting for tyrosine phosphorylated PECAM-1 and VEGFR2. Knockdown of PIEZO1 was verified by anti-PIEZO1 immunoblotting. Bar diagrams show the densitometric evaluation. (E) Concentration of ATP in the supernatant of HUAECs. Shown are means $\pm \mathrm{SEM} ;{ }^{*} P \leq 0.05 ;{ }^{* *} P \leq 0.01$ (2-way ANOVA and Bonferroni's post hoc test).

AKT (at serine 473) and eNOS (at serine 1177) in response to flow (Figure 1B). Induction of $\mathrm{Ca}^{2+}$ transients and of AKT and eNOS phosphorylation by carbachol acting through muscarinic $G_{q} / G_{11}$ coupled $\mathrm{M}_{3}$ receptors was not affected by PIEZO1 knockdown (Supplemental Figure 1, A and B; supplemental material available online with this article; doi:10.1172/JCI87343DS1), indicating that the cells had not lost the ability to respond to other stimuli. Knockdown efficiency of PIEZO1 in HUAECs was more than 95\% (Supplemental Figure 1C), and all effects could be verified by an independent siRNA as well as in bovine aortic endothelial cells (BAECs) (Supplemental Figure 1, D-F) and human umbilical vein endothelial cells (HUVECs) (Supplemental Figure 2, A and B). Consistent with a role of PIEZO1 in flow-induced eNOS phosphorylation, knockdown of PIEZO1 also strongly reduced flowinduced increases in nitrate levels in supernatants of HUAECs (Figure 1C), indicating a reduction in eNOS activity. Similarly, the fluid shear stress-induced phosphorylation of Src kinase, PECAM-1, and VEGFR2 was strongly reduced after knockdown of PIEZO1 (Figure 1D). Since flow-induced ATP release from endothelial cells and subsequent activation of the $G_{q} / G_{11}$-coupled P2Y receptor has been shown to be upstream of AKT and eNOS acti- vation in flow-induced endothelial responses (17), we tested the effect of a PIEZO1 knockdown on flow-induced ATP release. ATP release in response to flow was strongly reduced after knockdown of PIEZO1 in endothelial cells (Figure 1E), indicating that PIEZO1 operates upstream of ATP release in flow-induced effects.

Activation of PIEZO1 by Yoda1 mimics fluid shear stress-induced endothelial activation. We next tested whether the recently described specific activator of PIEZO1, Yoda1 $(25,26)$, was able to induce effects similar to those mediated by PIEZO1 in response to flow. As shown in Figure 2A and Supplemental Figure 3A, Yoda1 dosedependently increased $\left[\mathrm{Ca}^{2+}\right]_{\mathrm{i}}$, which was strongly reduced after knockdown of PIEZO1. Similarly, Yoda1 induced AKT and eNOS phosphorylation, increased nitrate formation, and caused an increase in ATP release (Figure 2, B-D), and all effects were strongly reduced after knockdown of PIEZO1. To test whether PIEZO1 indeed acts upstream of ATP release and subsequent activation of the $G_{q} / G_{11}$-coupled P2Y receptor, we tested whether Yoda1-induced endothelial effects were affected by the ATP-degrading enzyme apyrase and knockdown or blockade of $\mathrm{G} \alpha_{\mathrm{q}} / \mathrm{G} \alpha_{11}$ and $\mathrm{P} 2 \mathrm{Y}_{2}$. As shown in Figure 3, A-C, knockdown of $\mathrm{P} 2 \mathrm{Y}_{2}$ or $\mathrm{G} \alpha_{\mathrm{q}} / \mathrm{G} \alpha_{11}$ or incubation of cells with apyrase or the $\mathrm{P} 2 \mathrm{Y}_{2}$ receptor antagonist AR-C118925 strongly 
A
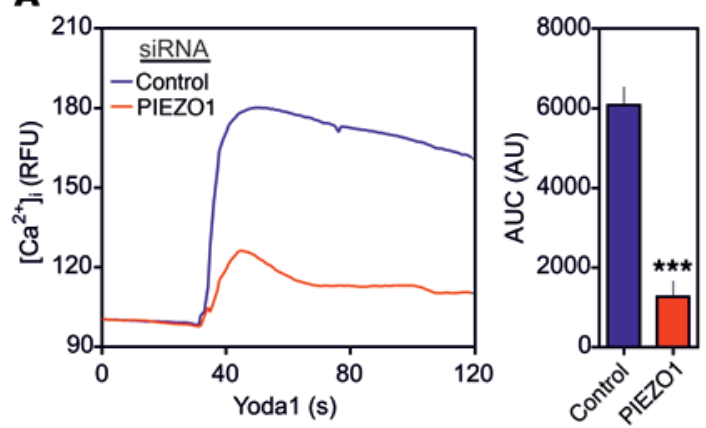

B
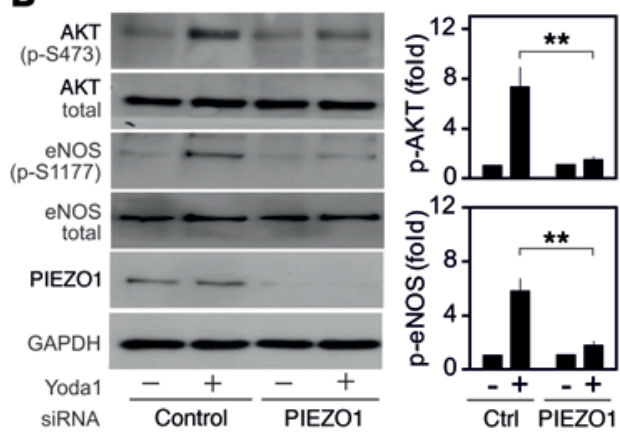

C
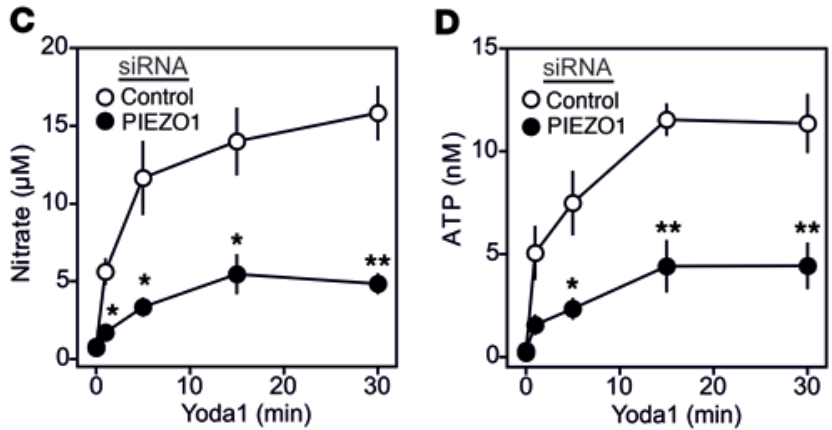

Figure 2. Yoda1 induces endothelial responses similar to fluid shear stress via PIEZ01. Cells were transfected with scrambled (control) siRNA or siRNA directed against PIEZO1. (A) Fluo-4-loaded HUAECs ( $n=24$, control; $n=22$, PIEZO1 knockdown; 3 independent experiments) were exposed to $1 \mu \mathrm{M}$ Yoda1, and $\left[\mathrm{Ca}^{2+}\right]_{i}$ was determined as fluorescence intensity (RFU, relative fluorescence units). Bar diagrams show AUC. Shown are means $\pm \mathrm{SEM}$; ${ }^{* * *} P \leq 0.001$ (2-tailed Student's $t$ test). (B-D) HUAECs were exposed to $1 \mu \mathrm{M}$ Yoda1 for the indicated times (5 minutes in B). AKT and eNOS activation was determined by Western blotting for phosphorylated AKT and eNOS as well as total AKT and eNOS $(n=3)$. Knockdown of PIEZO1 was verified by anti-PIEZO1 immunoblotting. Bar diagrams show the densitometric evaluation. ( $\mathbf{C}$ and $\mathbf{D})$ Nitrate concentration $(\mathbf{C}, n=3)$ and concentration of ATP $(\mathbf{D}, n=6)$ in the cell medium. Shown are the mean \pm SEM. ${ }^{*} P \leq 0.05$ and ${ }^{* *} P \leq 0.01$ (2-way ANOVA and Bonferroni's post-hoc test).

inhibited Yoda1-induced $\mathrm{Ca}^{2+}$ transients as well as AKT and eNOS phosphorylation. In addition, Yoda1-induced nitrate formation was strongly reduced after knockdown of $\mathrm{P} 2 \mathrm{Y}_{2}$ or $\mathrm{G} \alpha_{q} / \mathrm{G}_{11}$ (Figure 3D). These data indicate that $P 2 \mathrm{Y}_{2}$ and $\mathrm{G}_{\mathrm{q}} / \mathrm{G}_{11}$ are downstream of PIEZO1 in flow-induced endothelial signaling.

Pannexins mediate in part PIEZO1-dependent endothelial ATP release. We next analyzed the mechanisms linking PIEZO1-induced $\mathrm{Ca}^{2+}$ influx with the release of ATP. Since $\mathrm{Ca}^{2+}$ can activate pannexin channels (27), and since pannexin 1 has been shown to be involved in thrombin-induced, $\mathrm{Ca}^{2+}$-mediated ATP release from endothelial cells (28), we focused on pannexin channels. It has been shown that pannexin 1 is the predominant form of pannexins in endothelial cells (29). However, we also found expression of pannexin 2 (Supplemental Figure 3B), and therefore we tested the effect of a knockdown of both pannexin 1 and pannexin 2 on fluid shear stress- and Yoda1-induced endothelial ATP release and AKT and eNOS phosphorylation as well as on nitrate formation (Figure 4, A-F). In all cases we saw a significant but only partial reduction of flow- and Yoda1-induced effects, although the knockdown efficiency was very high (Supplemental Figure 3B). We conclude from this that pannexin channels are involved in PIEZO1-mediated, flow-induced ATP release but that other mechanisms contribute to this effect as well.

PIEZO1 mediates endothelial flow-induced vasodilation and controls vascular tone and blood pressure. To further explore the role of endothelial PIEZO1 in flow-induced vascular effects, we generated inducible endothelium-specific PIEZO1-deficient mice (Tie2-CreER ${ }^{T 2}$ Piezo1 $^{f / f l}$, hereafter referred to as EC-PIEZO1-KO mice). Induction of PIEZO1 deficiency in endothelial cells of EC-PIEZO1-KO mice was verified by RT-PCR and immunoblotting (Supplemental Figure 3, C and D). While precontracted mesenteric arteries from WT mice responded to an increase in flow with strong vasodilatation, arteries from induced EC-PIEZO1-KO mice had almost completely lost the ability to respond to flow (Figure 5A). This was not due to a general defect of the vessels from EC-PIEZO1-KO mice, as they relaxed and contracted normally to acetylcholine and phenylephrine, respectively (Figure $5 B)$. We then tested whether Yoda1 mimicked the effect of flow in isolated and perfused mesenteric arteries. As shown in Figure 5, C-E, precontracted WT mesenteric arteries were dilated after intravascular addition of Yoda1 at increasing concentrations. The effect of Yoda1 was strongly inhibited in mesenteric arteries from EC-PIEZO1-KO mice as well as from mice with endotheliumspecific loss of $\mathrm{G} \alpha_{\mathrm{q}} / \mathrm{G} \alpha_{11}(\mathrm{EC}-\mathrm{q} / 11-\mathrm{KO})(17)$. Also, mesenteric arteries from mice with endothelium-specific and induced $\mathrm{P}_{2} \mathrm{Y}_{2}$ deficiency (EC-P2Y2-KO) showed hardly any response to Yoda1 after precontraction, whereas mesenteric arteries from mice with endotheliumspecific pannexin 1/pannexin 2 double deficiency showed slightly reduced responses compared with WT vessels (Figure 5, C-E).

Since flow-induced vasodilation is regarded as one of the basic mechanisms contributing to vascular tone and basal blood pressure $(1,30)$, we analyzed the effect of an acute induction of endothelial PIEZO1 deficiency on arterial blood pressure in mice. As shown in Figure 6A, treatment of WT mice with tamoxifen resulted in a small and transient effect on the mean arterial blood pressure, whereas induction of endothelial PIEZO1 deficiency in EC-PIEZO1-KO mice by tamoxifen led to a significant increase of 10-15 mmHg within a few days (Figure 6A). In mice pretreated with angiotensin II or L-NAME to induce hypertension, tamoxifeninduced loss of endothelial PIEZO1 did not further increase blood pressure (Supplemental Figure 4, A and B). Again, a relatively minor but still significant increase in blood pressure was seen in tamoxifen-induced pannexin 1/pannexin 2 endothelium-specific double-knockout mice (Supplemental Figure 4C). The increase in mean arterial blood pressure after induction of endotheliumspecific PIEZO1 deficiency was accompanied by a decrease in plasma nitrate levels (Figure 6B) as well as by a reduced phosphor- 
A
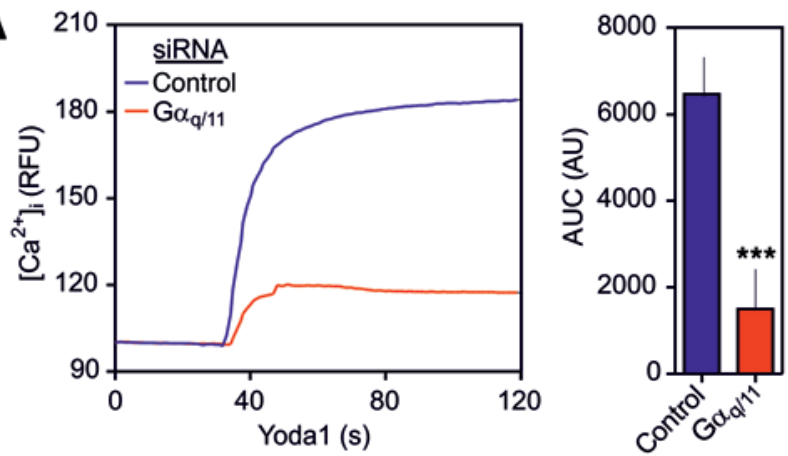

B
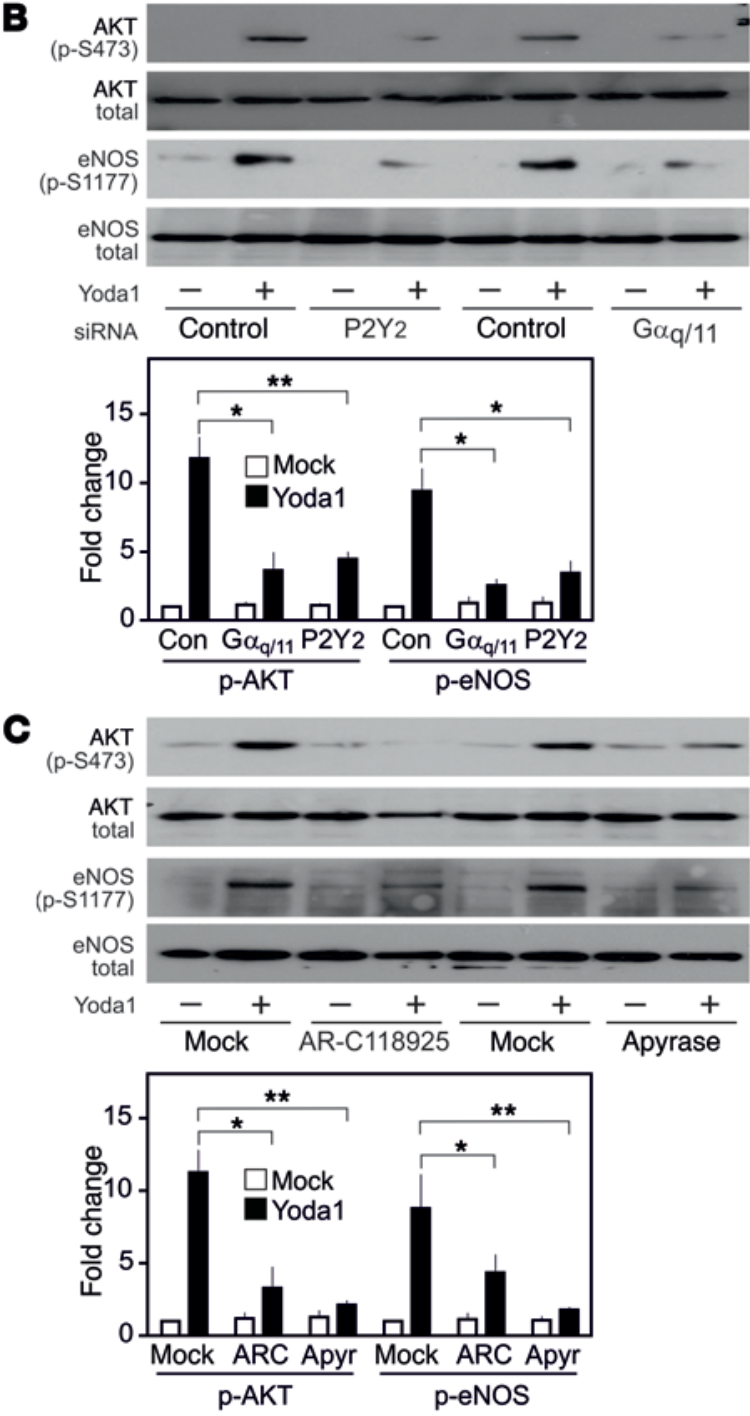

D

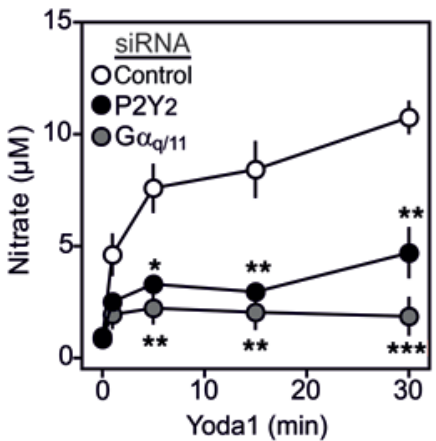

Figure 3. Yoda1-induced, PIEZ01-mediated endothelial effects involve P2Y and $\mathbf{G}_{q} / \mathbf{G}_{11}$ (A-D) HUAECs were transfected with scrambled siRNA (control; con) or siRNA directed against $P 2 Y_{2}$ or $G \alpha_{q} / G \alpha_{11}(\mathbf{A}, \mathbf{B}$, and $\mathbf{D}$ ) or were preincubated without or with $30 \mu \mathrm{M}$ of the $\mathrm{P} 2 \mathrm{Y}_{2}$ antagonist $A R-C 118925$ (ARC) or $2 \mathrm{U} / \mathrm{ml}$ apyrase (apyr) for 30 minutes (C). (A) Fluo-4-loaded HUAECs ( $n=14$, control; $n=20, G \alpha_{1 / 1}$ knockdown; 3 independent experiments) were exposed to $1 \mu \mathrm{M}$ Yoda1, and $\left[\mathrm{Ca}^{2+}\right]_{i}$ was determined as fluorescence intensity (RFU, relative fluorescence units). Bar diagrams show AUC. Shown are means $\pm \mathrm{SEM} ;{ }^{* *} P \leq 0.001$ (2-tailed Student's $t$ test). (B and C) Cells were exposed to $1 \mu \mathrm{M}$ Yoda1 for 5 minutes, and AKT and eNOS activation was determined as described. Bar diagrams show the densitometric evaluation $(n=3)$. (D) Nitrate concentration in the cell medium $(n=4)$. Shown are the mean \pm SEM. ${ }^{*} P \leq 0.05,{ }^{*} P \leq 0.01$, and ${ }^{* *} P \leq 0.001$ (2-way ANOVA and Bonferroni's post-hoc test).

ylation of the murine mesenteric artery eNOS at serine 1176 (Figure 6C). These data clearly show that endothelial PIEZO1-mediated signaling is a central regulatory mechanism controlling endothelial NO formation, vascular tone, and arterial blood pressure in vivo.

\section{Discussion}

Arterial blood pressure is controlled under normal and hypertensive conditions by various vasodilatory factors, which are released from the endothelium in response to fluid shear stress exerted by flowing blood $(3-5,7)$. These factors include NO, endotheliumderived hyperpolarizing factor (EDHF), and prostanoids $(31,32)$. Overall, NO appears to play a predominant role also for systemic blood pressure regulation, and several mechanotransducing signaling pathways involved in fluid shear stress-induced stimulation of NO formation have been identified $(1,2,6)$. More recently, evidence has been provided that flow-induced endothelial release of ATP is a critical upstream step in the mechanotransducing signaling cascade, resulting in NO formation and vascular relaxation (17, 33, 34). The ATP released in response to flow acts through the $G_{q} / G_{11}$ coupled $\mathrm{P} 2 \mathrm{Y}_{2}$ receptor, resulting in $\mathrm{PECAM}-1 / \mathrm{VE}$-cadherin/ VEGFR2-mediated activation of AKT and subsequent NO formation via eNOS (17). How fluid shear stress induces ATP release and activation of $\mathrm{P} 2 \mathrm{Y}_{2} / \mathrm{G}_{\mathrm{q}} / \mathrm{G}_{11}$-mediated signaling is, however, unclear. Here we show that the endothelial mechanosensitive cation channel PIEZO1 is required for flow-induced ATP release and subsequent $P 2 Y_{2} / G_{q} / G_{11}$-mediated activation of downstream signaling resulting in phosphorylation of AKT and eNOS. The PIEZO1-dependent ATP release is in part mediated by pannexin channels. We also show that endothelial PIEZO1 is required for flow-induced NO formation by eNOS and for flow-induced vasodilatation, as well as for maintaining basal blood pressure. The specific PIEZO1 activator Yoda1 fully mimicked the effect of fluid shear stress on endothelial cells.

While PIEZO proteins have been shown to be involved in mechanosensing in various biological processes, the precise physical stimulus and activation mechanism that results in the opening of the channel pore of PIEZO is still unclear. It is possible that the PIEZO1 channel is gated by forces transferred to the channel protein by direct interaction with the cytoskeleton or with the extracellular matrix. An involvement of cytoskeletal structures in mechanosensing of fluid shear stress in endothelial cells has been suggested based on the observation that inhibition of cytoskeletal filaments blocks various endothelial responses to flow $(1,35)$. However, so far no interaction 
A

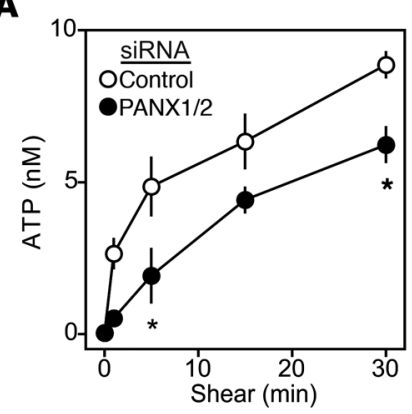

D

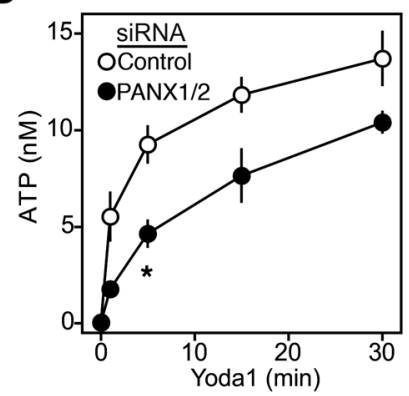

B

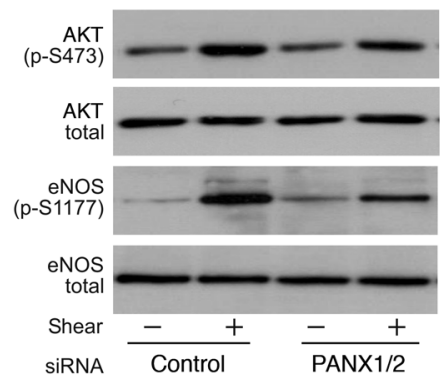

E

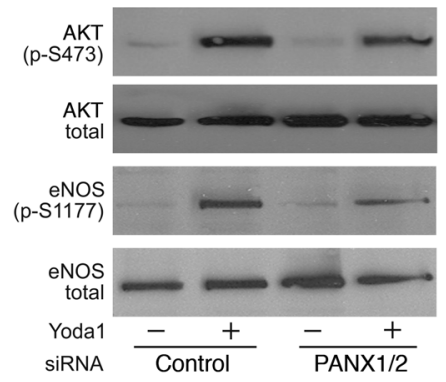

C
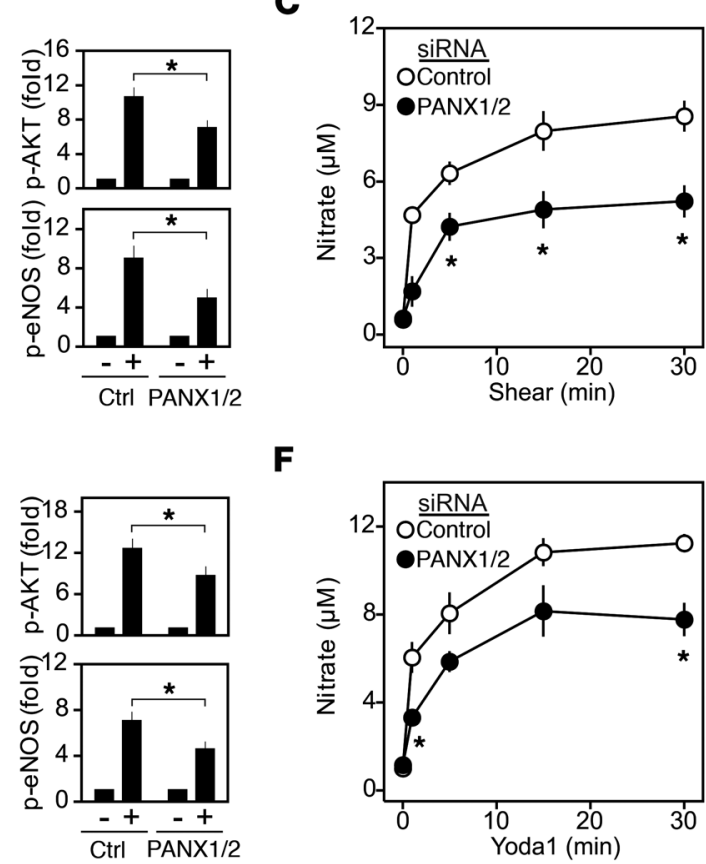

$\mathbf{F}$

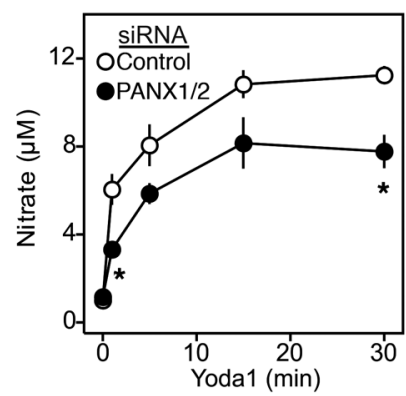

Figure 4. Role of pannexins in flow- and Yoda1-induced endothelial effects. (A-F) HUAECs were transfected with scrambled (control) siRNA or siRNAs directed against pannexin 1 and pannexin 2 (PANX1/2) and were exposed to fluid shear (15 dyn/cm²) (A-C) or $1 \mu M$ Yoda1 (D-F) for the indicated time periods (5 minutes in $\mathbf{B}$ and $\mathbf{E})$. The concentration of ATP ( $\mathbf{A}$ and $\mathbf{D}, n=3)$ or nitrate $(\mathbf{C}$ and $\mathbf{F}, n=4)$ in the cell medium was measured. AKT and eNOS activation (B and $\mathbf{E}, n=3$ ) was determined by Western blotting for phosphorylated AKT and eNOS as well as total AKT and eNOS. Bar diagrams show the densitometric evaluation. Shown are the mean \pm SEM. ${ }^{*} P \leq 0.05$ (2-way ANOVA and Bonferroni's post-hoc test).

of PIEZO1 with cytoskeletal structures or the extracellular matrix has been described. It is also possible that accessory proteins of PIEZO1 tether the channel to the cellular or extracellular matrix, or modulate the sensitivity of PIEZO1 to the mechanical stimulus. The latter mechanism has been shown to operate in touch-sensitive cells in which PIEZO interacts with stomatin-like protein 3 (STOML3), which modulates PIEZO channels and greatly increases PIEZO sensitivity (36). The generation of lateral membrane tension resulting from the fluid shear stress on the apical surface of the endothelial cell layer has been proposed to be one of the primary effects of endothelial fluid shear stress $(37,38)$. Interestingly, PIEZO1 has been found to be, in particular, sensitive to membrane bilayer tension $(39,40)$, which therefore would be consistent with a role of PIEZO1 in direct endothelial flow sensing.

It has been described that only a minor fraction of the $\left[\mathrm{Ca}^{2+}\right]_{i}$ elevation induced through activation of PIEZO1 is due to PIEZO1mediated influx and that the majority of the $\mathrm{Ca}^{2+}$ signal results from downstream amplification mechanisms $(41,42)$. Consistent with this, we found that endothelial loss of $G_{q} / G_{11}$ strongly inhibited flow-induced increases in $\left[\mathrm{Ca}^{2+}\right]_{i}(17)$. We also used the only recently developed PIEZO1 activator, Yoda1 $(25,26)$, to simulate flowdependent PIEZO1 activation. Yoda1 appeared to be a rather specific PIEZO1 activator, as effects induced in endothelial cells as well as in vessels were strongly reduced after knockdown or endothelial knockout of PIEZO1. Again, the majority of the increase in $\left[\mathrm{Ca}^{2+}\right]_{\mathrm{i}}$ induced by direct PIEZO1 activation by Yoda1 depended on $\mathrm{G}_{\mathrm{q}} / \mathrm{G}_{11}$ mediated signaling. In contrast, when we activated $G_{q} / G_{11}$ through a $G_{q} / G_{11}$-coupled receptor such as muscarinic receptors by carbachol, knockdown of PIEZO1 did not affect carbachol- induced calcium transients. This strongly indicates that PIEZO1 is upstream of $\mathrm{G}_{\mathrm{q}} / \mathrm{G}_{11}$-mediated signaling and that the contribution of PIEZO1-mediated $\mathrm{Ca}^{2+}$ influx to the overall flow-induced increase in intracellular free calcium is relatively small compared with the $\mathrm{G}_{\mathrm{q}} / \mathrm{G}_{11}$-mediated portion. While this transient $\left[\mathrm{Ca}^{2+}\right]_{\mathrm{i}}$ elevation in response to an acute increase in flow can lead to a $\mathrm{Ca}^{2+}$ / calmodulin-mediated activation of eNOS $(6,8,9)$, sustained NO formation has been shown to require phosphorylation of eNOS by protein kinases such as AKT or other kinases $(6,10-12)$. The latter mechanism, which involves the mechanosensory complex consisting of PECAM-1, VE-cadherin, and VEGFR2/VEGFR3 (13-16), is probably the predominant way in which PIEZO1 and $\mathrm{G}_{\mathrm{q}} / \mathrm{G}_{11}$ mediate the flow-induced activation of NO formation in the context of long-term regulation of vascular tone and blood pressure.

Much evidence has been provided that increased shear stress on endothelial cells induces the release of ATP (17, 33, $34,43,44)$. However, the mechanism underlying the release of ATP from endothelial cells is still poorly understood, and several mechanisms, including vesicular exocytosis, ABC transportermediated release, or release through connexin hemichannels or pannexin channels, have been suggested (45). Our data clearly indicate that PIEZO1 mediates shear stress-induced ATP release. This strongly suggests that the intracellular increase in $\mathrm{Ca}^{2+}$ concentration resulting from PIEZO1 activation plays a critical role in ATP release. Consistent with this, an increase in intracellular $\mathrm{Ca}^{2+}$ concentration has been shown to induce endothelial ATP release (46), and to mediate ATP release from endothelial cells in response to thrombin (28). Interestingly, pannexin channels can be activated by increased intracellular $\mathrm{Ca}^{2+}$ concentra- 
A

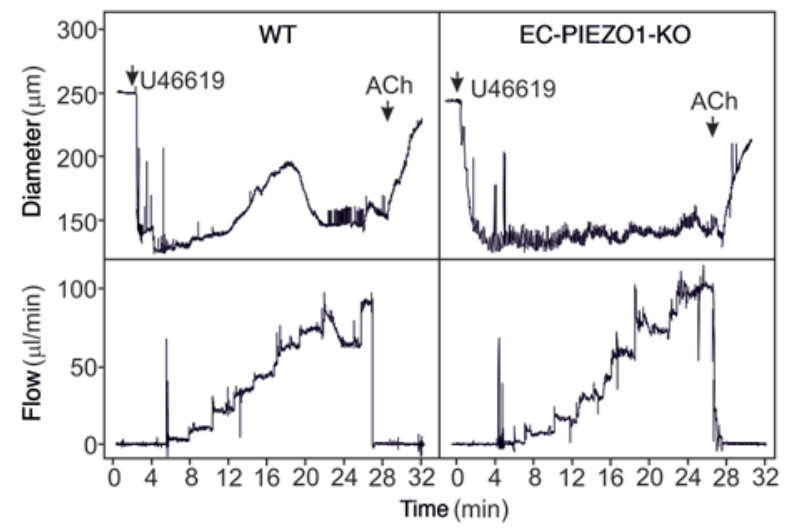

C

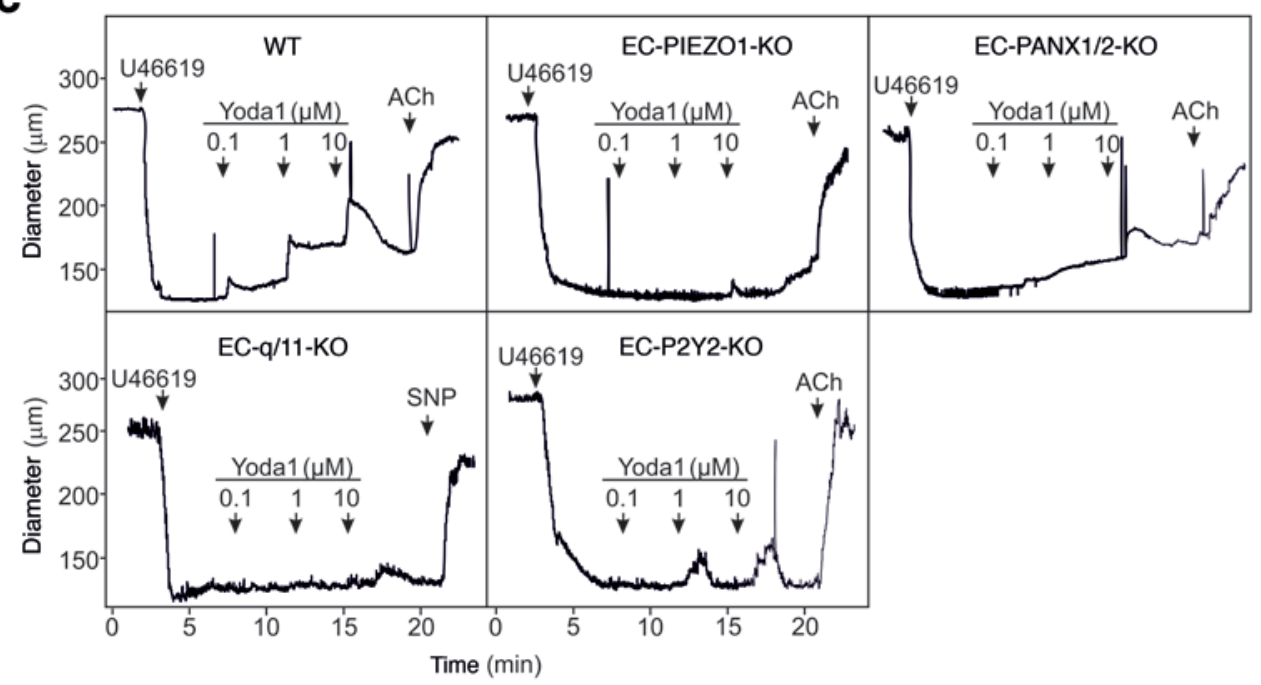

B

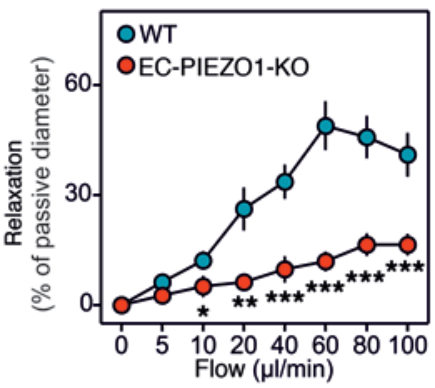

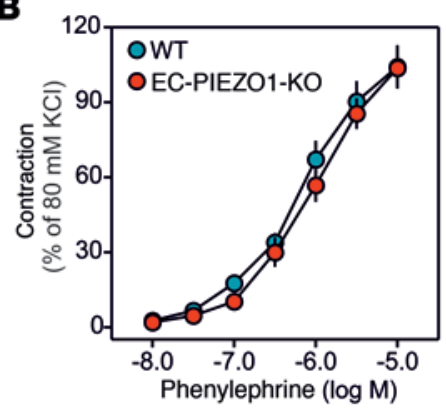

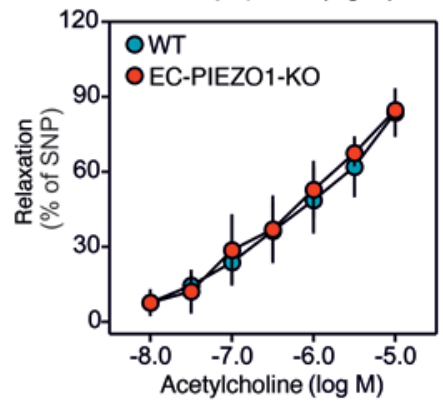

D

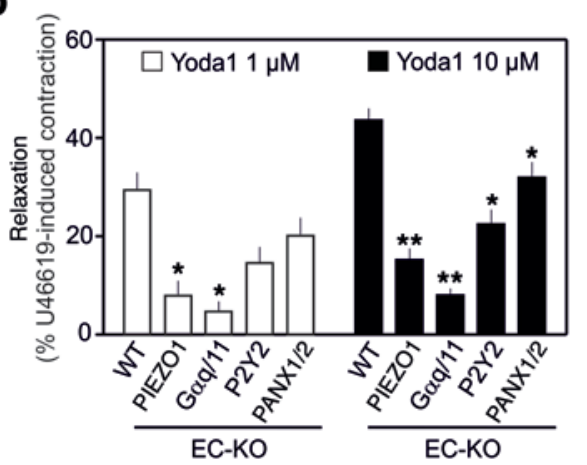

$\mathbf{E}$

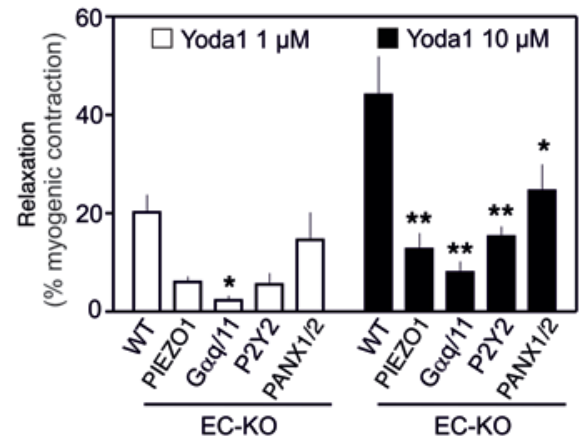

Figure 5. Endothelial PIEZO1 is required for flow-induced vasodilation. (A) Effect of a stepwise increase in perfusion flow on the diameter of mesenteric arteries from tamoxifen-treated WT mice or EC-PIEZO1-KO mice precontracted with $100 \mathrm{nM}$ of the thromboxane $\mathrm{A}_{2}$ analog U46619. After flow was stopped, $10 \mu \mathrm{M}$ acetylcholine (ACh) was added. Right panel: Flow-induced vasorelaxation as percentage of the passive vessel diameter ( $n=6$, WT; $n=7$, EC-PIEZO1-KO). (B) Effect of acetylcholine and phenylephrine on the tension of mesenteric artery stripes from WT $(n=5)$ and EC-PIEZO1-KO animals ( $n=5)$. (C-E) Effect of Yoda1 at the indicated concentration on the diameter of mesenteric arteries precontracted by U46619 (C and $\mathbf{D})$ or by induction of myogenic tone (E) from WT $(n=4)$, EC-PIEZO1-KO $(n=4)$, EC-q/11-KO $(n=3)$, EC-P2Y2-KO $(n=3)$, or EC-PANX1/2-KO $(n=3)$ mice. Vessels were prepared 7-10 days after tamoxifendependent induction. Shown are means \pm SEM. ${ }^{*} P \leq 0.05,{ }^{* *} P \leq 0.01$, and ${ }^{* * *} P \leq 0.001$ (2-way ANOVA and Bonferroni's post-hoc test).

tions (27), and evidence has been provided that $\mathrm{Ca}^{2+}$-mediated endothelial ATP release involves pannexin 1, the major pannexin channel expressed in endothelial cells $(28,29,47)$. Our in vitro and in vivo data show that pannexin is indeed involved in flowinduced endothelial ATP release and other PIEZO1-mediated in vitro and in vivo effects. However, in none of the in vitro and in vivo models did loss of endothelial pannexin channels completely block PIEZO1-dependent effects, indicating that pannexin channels only partially account for the observed endothelial ATP release and that additional mechanisms are involved. Recent 
A

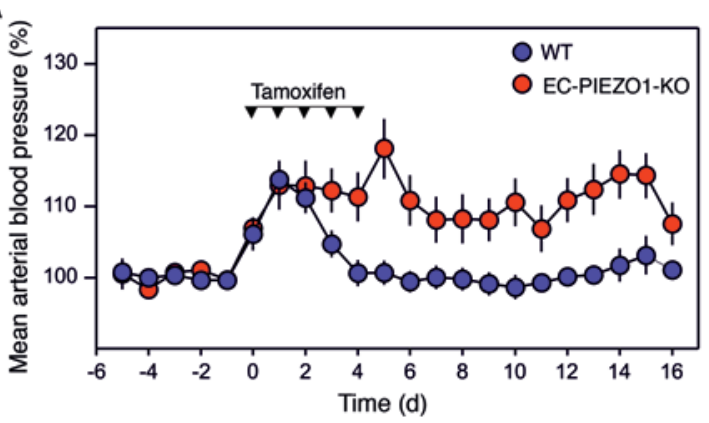

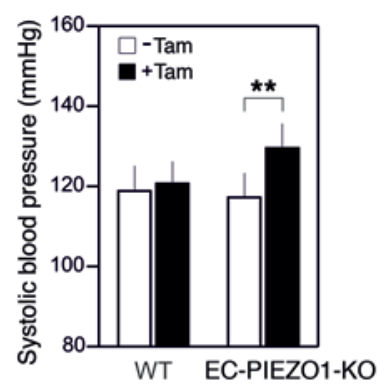

B

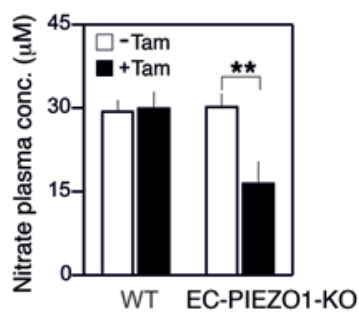

\section{C}
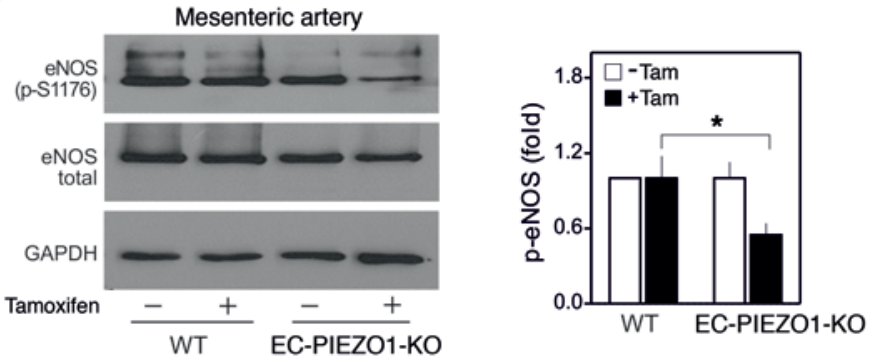

Figure 6. Endothelial PIEZO1 controls blood pressure. (A) Blood pressure in WT ( $n=12)$ and EC-PIEZO1-KO mice $(n=14)$ before, during, and after induction by tamoxifen. Average blood pressure 5 days before induction was set at $100 \%$. The bar diagram shows systolic arterial blood pressure 4 days before tamoxifen treatment and in the second week after induction. (B) Plasma nitrate levels in WT $(n=8)$ and EC-PIEZO1-KO mice ( $n=8)$ before and 5 days after induction. (C) Phosphorylation of eNOS at S1176 in lysates of mesenteric arteries prepared 3 days after induction from WT and EC-PIEZO1-KO mice. Bar diagram shows a densitometric evaluation $(n=4)$. Shown are the mean \pm SEM. ${ }^{*} P \leq 0.05$ and ${ }^{* *} P \leq 0.01$ ( 2 -tailed Student's $t$ test, B and C, and 2 -way ANOVA and Bonferroni's post-hoc test, A).

studies using urothelial cells and red blood cells have also shown a PIEZO1-mediated mechanotransductive release of ATP, indicating that this is a cellular mechanism not restricted to endothelial cells $(48,49)$. However, the process of PIEZO1-mediated ATP release from endothelial cells appears to involve additional mechanisms that still need to be fully explored.

The fact that an acute loss of endothelial PIEZO1-mediated mechanosignaling reduces eNOS activity in vivo, resulting in arterial hypertension, shows that the mechanosensing activity of PIEZO1 is constantly operating and is required for the basal fluid shear stress-induced NO formation and vasodilation. Defects in PIEZO1 function are likely to cause alterations in vascular development and regulation, including arterial hypertension. In fact, biallelic mutations in PIEZO1 resulting in attenuated PIEZO1 function have recently been described in patients with congenital lymphatic dysplasia (50). It will be interesting to analyze the function and structure of PIEZO1 in various forms of arterial hypertension. At the same time, PIEZO1 is also a potential target for preventive or therapeutic interventions aiming at an alteration of vascular tone. The strong vasodilatory effect of Yoda1, which is fully PIEZO1dependent, suggests that a PIEZO1 activator can not only reduce vascular tone and elevated blood pressure but may also improve endothelial function by increasing endothelial $\mathrm{NO}$ formation. It will be interesting to test the effect of an activation of PIEZO1 in various models of arterial hypertension or endothelial dysfunction.

\section{Methods}

Reagents. Acetylcholine, carbachol, phenylephrine, and tamoxifen were from Sigma-Aldrich. Insulin was from Gibco. Yoda1 was from Maybridge (catalog SPB07298). Apyrase was from New England BioLabs (catalog M0393L). ARL 67156 was from Tocris (catalog 1283). The ${\mathrm{P} 2 \mathrm{Y}_{2}}_{\text {receptor }}$ antagonist AR-C118925 was provided by Christa E. Müller (PharmaCenter Bonn, Pharmaceutical Institute, Bonn, Germany) (17). U46619 was from Cayman Chemical. Anti-phospho-eNOS (S1177, human; catalog 9571), anti-phospho-AKT (S473; catalog 4060), anti-AKT (catalog 9272), anti-GAPDH (catalog 2118), anti-phospho-Src kinase (Y416; catalog 6943), anti-Src (catalog 2109), and anti-VEGFR-2 (catalog 2479) antibodies were from Cell Signaling. Anti-phosphotyrosine antibody (4G10; catalog 05-1050X) was from Millipore. Anti-eNOS antibody was from BD Biosciences (catalog 610296). Anti-PIEZO1 antibody was from Proteintech (catalog 15939-1-AP). Anti-G $\alpha_{q} / G \alpha_{11}$ (catalog sc-392) and anti-PECAM-1 (catalog sc-1506) antibodies were from Santa Cruz Biotechnology. Anti-CD31 (catalog 13-0311-82), antiCD144-PE (catalog 12-1441-82), and anti-Ter-119 antibodies (catalog 14-5921-82) were from eBioscience.

Primary cells. HUAECs, BAECs, and HUVECs were obtained from Lonza. HUAECs and BAECs were cultured with endothelial growth medium (EGM-2-MV, Lonza), and HUVECs were cultured with EGM-2 (Lonza). Confluent cells at passage 3 or less were used in experiments.

siRNA-mediated knockdown. Cells were transfected with siRNAs using Opti-MEM and Lipofectamine RNAiMAX (Invitrogen) as described previously (17). For transfection of cells in ibidi flow chambers, 5 pmol of siRNA was mixed gently with RNAiMAX in $20 \mu \mathrm{l}$ and incubated for 30 minutes at room temperature. The mixture was added to $100 \mu \mathrm{l}$ cell culture medium, and cells in the ibidi chamber $\left(2.5 \mathrm{~cm}^{2}\right)$ were covered by the medium. Medium was changed after 6 hours. Eighteen hours later, the siRNA transfection was repeated, and experiments were performed after 48 hours after second siRNA transfection. For transfection of cells 
in 33- $\mathrm{cm}^{2}$ dishes from the BioTech-Flow System cone-plate viscosimeter (see below), 50 pmol siRNA in $100 \mu \mathrm{l}$ was prepared and added to $1.5 \mathrm{ml}$ cell culture medium. siRNAs against PIEZO1, pannexin 1, pannexin 2, $G \alpha_{q}, G \alpha_{11}$, and P2Y $Y_{2}$ were from Qiagen or Sigma-Aldrich. The targeted sequences of siRNAs directed against RNAs encoding $\mathrm{G} \alpha_{\mathrm{q}}, \mathrm{G} \alpha_{11}, \mathrm{P} 2 \mathrm{Y}_{2}$, PIEZO1, pannexin 1, and pannexin 2 were: GNAQ (human), 5'-CAGGACACATCGTTCGATTTA-3' and 5'-CAGGAATGCTATGATAGACGA-3'; GNA11 (human), 5'-CTACAAGTACGAGCAGAACAA-3' and 5'-AACGTGACATCCATCATGTTT-3'; P2RY2 (human), 5'-CCCTTCAGCACGGTGCTCT-3', 5'-CTGCCTAGGGCCAAGCGCA-3', and 5'-GCTCGTACGCTTTGCCCGA-3'; PIEZO1 (human), 5'-CCAAGTACTGGATCTATGT-3', 5'-GCAAGTTCGTGCGCGGATT-3', and 5'-AGAAGAAGATCGTCAAGTA-3'; PIEZO1 (bovine), 5'-GTGTTTGGTCTCAAGGACT-3' and 5'-GTGTCTACTTCCTGCTCTT-3'; pannexin 1 (human), 5'-CATTTAATCGTCAAGTACA-3' and 5'-CTTAAGGTCCTGGAGAATA-3'; pannexin 2 (human), 5'-CGCTCAACCGGCTGGACTT-3' and 5'-CGCAGAAGCAGAACGAGTT-3'.

Determination of $\left[\mathrm{Ca}^{2+}\right]_{i}$. For the determination of the intracellular $\mathrm{Ca}^{2+}$ concentration, cells were placed in a microfluidic plate (Fluxion) and loaded with Fluo-4 AM (Molecular Probes). Laminar shear stress was generated using the BioFlux200 system (Fluxion), and live cell images were acquired with an Olympus IX81 microscope.

Shear stress assays. For biochemical experiments to determine protein phosphorylation by immunoblotting, cells were exposed to flow using a parallel-plate flow chamber ( $\mu$-Slide I 0.4 Luer, ibidi). The flow chamber was perfused with a constant flow rate using a computercontrolled air pressure pump (ibidi). The constant wall shear stress $(\tau)$ is given by $\tau=\eta \times 131.6 \varphi ; \eta$ : viscosity of the medium; $\varphi$ : flow rate (according to the manufacturer's instructions).

For immunoprecipitation experiments and experiments to determine nitrate and ATP levels, the BioTech-Flow System cone-plate viscosimeter (MOS Technologies) was used to expose cells to fluid shear stress as described previously (51). The cone of this system has an angle of $2.5^{\circ}$ and rotates on top of a $33-\mathrm{cm}^{2}$ cell culture dish containing $3 \mathrm{ml}$ of medium. Shear stress was calculated with the following formula (assuming a Reynolds number of $<<1$ ): $\tau=\eta \times 2 \pi n / 0.044$ ( $\tau$ : shear stress; $\eta$ : viscosity; $n$ : rotational speed). For application of arterial shear stress at $15 \mathrm{dyn} / \mathrm{cm}^{2}, 3 \%(\mathrm{wt} / \mathrm{vol}$ ) polyvinylpyrrolidone was added to the cell culture medium to increase the viscosity $(\eta)$ to $0.00635 \mathrm{dyn} / \mathrm{cm}^{2}$, and a rotation speed of $100 \mathrm{rpm}(1.666 \mathrm{r} / \mathrm{s})$ was used.

Western blotting and immunoprecipitation. Cells were lysed in Triton X-100 buffer (Cell Signaling, 9803) supplemented with protease and phosphatase inhibitors (Cell Signaling, 5872), and isolated mesenteric arteries were lysed by RIPA buffer (Cell Signaling, 9806) supplemented with protease and phosphatase inhibitors (see above). Lysates were centrifuged at 10,000 $\mathrm{g}$ for 10 minutes. For immunoprecipitation experiments, soluble supernatants were incubated with appropriate antibodies and protein A/G PLUS-agarose (Santa Cruz Biotechnology) $(1 \mu \mathrm{g}$ antibody per $100 \mu \mathrm{g}$ protein and $10 \mu \mathrm{l}$ beads in $100 \mu \mathrm{l}$ lysis buffer) overnight at $4^{\circ} \mathrm{C}$. The beads were washed 5 times with lysis buffer (see above). Immunoprecipitates or total cell lysates were then subjected to SDS-PAGE and transferred to nitrocellulose membranes. Membranes were probed with primary and HRP-conjugated secondary antibodies (Cell Signaling Technology) and were developed using the ECL detection system (Pierce).

Determination of ATP concentration. Cells were seeded at a density of $8 \times 10^{3}$ cells $/ \mathrm{cm}^{2}$ in a flow chamber of the BioTech-Flow Sys- tem from MOS Technologies (see above). After reaching confluence, cells were kept under static conditions or were exposed to fluid shear stress using the BioTech-Flow System (see above) for up to $30 \mathrm{~min}$ utes, and the ATP concentration in the supernatant was determined at the indicated time points using a bioluminescence assay (Molecular Probes, catalog A22066) according to the manufacturer's instructions. To inhibit ATP hydrolysis, $30 \mu \mathrm{M}$ ectonucleotidase inhibitor 6- $N, N$-diethyl- $\beta$ - $\gamma$-dibromomethylene-D-adenosine-5-triphosphate FPL 67156 (ARL 67156 trisodium salt) was added to the extracellular solution. Luminescence intensity was measured with a Flexstation-3 (Molecular Devices). ATP amounts were calculated using a calibration curve constructed using ATP standards. Parallel determination of lactate hydrogenase (LDH) activity (Cayman Chemical, catalog 10008882) showed no LDH activity, excluding that cell damage contributed to the increased ATP release under fluid shear stress.

Determination of nitrate levels. For plasma nitrate assays, blood collected by venous puncture was immediately centrifuged at $6,800 \mathrm{~g}$ for 5 minutes at $4^{\circ} \mathrm{C}$, and $20 \mu \mathrm{l}$ plasma was used for nitrate measurement. To determine flow-induced nitrate release from endothelial cells, $8 \times 10^{3}$ cells $/ \mathrm{cm}^{2}$ were seeded in a flow chamber (MOS Technologies). After reaching confluence, cells were kept under static conditions or were exposed to fluid shear stress using the BioTech-Flow System (see above) for up to 30 minutes. One hundred microliters flow medium at different time points was taken for the nitrate measurement. Nitrate and nitrite levels in mouse plasma or cell supernatants were determined using a nitrate/nitrite fluorometric assay kit from Cayman Chemical according to the manufacturer's instructions.

Animal models. All mice were backcrossed onto a C57BL/6N background at least 8-10 times, and experiments were performed with littermates as controls. Male and female animals at an age of 8-12 weeks were used unless stated otherwise. Mice were housed under a 12-hour light-dark cycle with free access to food and water and under specific pathogen-free conditions unless stated otherwise. The generation of inducible endothelium-specific $\mathrm{G} \alpha_{\mathrm{q}} / \mathrm{Ga}_{11}$-deficient mice (Tie2-CreER ${ }^{T 2}$ Gnaq ${ }^{f / f l}$ Gna11-- $\left.[\mathrm{EC}-\mathrm{q} / 11-\mathrm{KO}]\right)$ and mice with inducible endothelial-specific deficiency of $\mathrm{P}_{2} \mathrm{Y}_{2}$ (EC-P2Y2-KO) was described previously $(17,52)$. Mice carrying floxed alleles of Piezo1 (Piezo1 ${ }^{\text {mla(KOMP)Wtsi), }}$

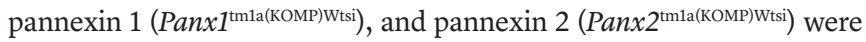
obtained from the Knockout Mouse Project (KOMP) Repository.

Telemetric blood pressure measurements. Measurements were performed in conscious, unrestrained mice with a radiotelemetry system (PA-C10, Data Sciences International) as described previously (53). Mice were caged with blinded identity and random orders.

Pressure myography. Pressure myography experiments were performed as described previously (17). Briefly, 7-10 days after tamoxifen injection, arteries of the third or fourth branch were removed from the mesentery and were mounted between 2 glass micropipettes seated in a chamber (Danish Myo Technology). The external diameter of the artery was visualized and recorded with a CCD camera using MyoView software. After reaching a steady-state diameter, vessels were contracted with $50-150 \mathrm{nM} \mathrm{U} 46619$ to $40 \%-50 \%$ of the passive diameter or by increasing of the intravascular pressure to 75-90 $\mathrm{mmHg}$ to induce myogenic contraction. After reaching a stable baseline, flow was increased in a stepwise manner by changing of the pressure of the inflow and outflow side inversely, thereby creating a pressure difference across the arteriole without altering the intraluminal pressure. Alternatively, Yoda1 was given into the perfu- 
sion buffer and was intraluminally delivered to the vessel by low flow ( $\leq 5 \mu \mathrm{l} / \mathrm{min}$ ). The viability of the vessel was verified at the end of the experiment by dilation of the vessel with acetylcholine $(10 \mu \mathrm{M})$ or sodium nitroprusside $(100 \mu \mathrm{M})$. Vasodilatation to flow or Yoda1 was calculated as a percentage of the U46619-induced contraction as described previously (54). Mice used for the experiments were caged with blinded identity and random orders.

Expression analysis. RNA isolation and transcription were performed as described previously (55). Primers were designed with the online tool provided by Roche, and quantification was performed using the LightCycler 480 Probe Master System (Roche). Genomic DNA was used as a universal standard to calculate gene copy number per nanogram of RNA. Relative expression levels were obtained by normalization with GAPDH or $18 S$ values. Primary mouse endothelial cells were isolated from skeletal muscle using CD31-labeled Dynabeads (Thermo Fisher Scientific) and further purification by CD144-PE labeling and FACS. Red blood cells were isolated by anti-Ter-119-labeled Dynabeads. RNA was prepared from sorted cells using Qiagen Micro Kit, and cDNA was preamplified using ProtoScript II Reverse Transcriptase (M0368S, New England BioLabs) for expression analysis by RT-PCR.

Quantitative PCR primer sequences. Quantitative PCR primer sequences were as follows: GNAQ (human), forward 5'-GACTACTTCCCAGAATATGATGGAC-3', reverse 5'-GGTTCAGGTCCACGAACATC-3'; GNA11 (human), forward 5'-GCATCCAGGAATGCTACGAC-3', reverse 5'-GGTCAACGTCGGTCAGGTAG-3'; GAPDH (human), forward 5'-GCATCCTGGGCTACACTGA-3', reverse 5'-CCAGCGTCAAAGGTGGAG-3'; Gapdh (mouse), forward 5'-AGCTTGTCATCAACGGGAAG-3', reverse 5'-TTTGATGTTAGTGGGGTCTCG-3'; PIEZO1 (human), forward 5'-CGTCTTCGTGGAGCAGATG-3', reverse 5'-GCCCTTGACGGTGCATAC-3'; PIEZO1 (bovine), forward 5'-TCAATGCTAACCACGACTGG-3', reverse 5'-GGTCACCGTGTAGCAGAGC-3'; Piezo1 (mouse), forward 5'-CCTTCTGTTGCTGGTGTTTG-3', reverse 5'-GTCCTGGTCCAACCTCTGG-3'; pannexin 1 (human), forward 5'-GATGGCAAAACTCCCATGTC-3', reverse 5'-AGCTCTGCCGTCTGGTTC-3'; pannexin 1 (mouse), forward 5'-GTGAGCTCAAATCGTACAAGTGTC-3', reverse 5'-CCTGCCCATTGCTTTTAATG-3'; pan- nexin 2 (human), forward 5'-ACAAGTTCCTGCCCTACGC-3', reverse 5'-AAGTTGAGCTCGGAGGTGAG-3'; pannexin 2 (mouse), forward 5'-CTGTGCCCCCTCAGACAT-3', reverse 5'-TGGCGATGAGGATCTGCT-3'; Cdh5 (encoding VE-cadherin; mouse), forward 5'-CCATGATCGACGTGAAGAAA-3', reverse 5'-GATGTGCAGTGTGTCGTATGG-3'.

Statistics. Trial experiments or experiments done previously were used to determine sample size with adequate statistical power. Samples were excluded in cases where RNA/cDNA quality or tissue quality after processing was poor (below commonly accepted standards). Data are presented as means \pm SEM. Comparisons between 2 groups were performed with unpaired 2-tailed Student's $t$ test, and multiple group comparisons at different time points were performed by 2-way ANOVA followed by Bonferroni's post hoc test. $P \leq 0.05$ was considered to be statistically significant.

Study approval. All procedures of animal care and use in this study were approved by the local animal ethics committees (Regierungspräsidium Karlsruhe and Darmstadt, Germany).

\section{Author contributions}

SW performed most of the in vitro and in vivo experiments, analyzed and discussed data, and commented on the manuscript. RC, $\mathrm{AI}$, and HK helped with pressure myography experiments, mouse generation, and expression analysis. NW initiated and supervised the study, and discussed data and commented on the manuscript. SO initiated and supervised the study, discussed data, and wrote the manuscript.

\section{Acknowledgments}

We thank Svea Hümmer for secretarial help. This work was supported by the Collaborative Research Center 834 of the German Research Foundation.

Address correspondence to: Stefan Offermanns, Max Planck Institute for Heart and Lung Research, Ludwigstrasse 43, $61231 \mathrm{Bad}$ Nauheim, Germany. Phone: 49.0.6032.705.1201; E-mail: stefan. offermanns@mpi-bn.mpg.de.
1. Hahn C, Schwartz MA. Mechanotransduction in vascular physiology and atherogenesis. Nat Rev Mol Cell Biol. 2009;10(1):53-62.

2. Tarbell JM, Simon SI, Curry FR. Mechanosensing at the vascular interface. Annu Rev Biomed Eng. 2014;16:505-532.

3. Davies PF. Flow-mediated endothelial mechanotransduction. Physiol Rev. 1995;75(3):519-560.

4. Félétou M, Köhler R, Vanhoutte PM. Endothelium-derived vasoactive factors and hypertension: possible roles in pathogenesis and as treatment targets. Curr Hypertens Rep. 2010;12(4):267-275.

5 . Busse R, Fleming I. Regulation of endotheliumderived vasoactive autacoid production by hemodynamic forces. Trends Pharmacol Sci. 2003;24(1):24-29.

6. Fleming I. Molecular mechanisms underlying the activation of eNOS. Pflugers Arch. 2010;459(6):793-806.

7. Zhou J, Li YS, Chien S. Shear stress-initiated signaling and its regulation of endothelial function. Arterioscler Thromb Vasc Biol. 2014;34(10):2191-2198.
8. Michel T, Vanhoutte PM. Cellular signaling and NO production. Pflugers Arch. 2010;459(6):807-816.

9. Kuchan MJ, Frangos JA. Role of calcium and calmodulin in flow-induced nitric oxide production in endothelial cells. Am J Physiol. 1994;266(3 pt 1):C628-C636.

10. Dimmeler S, Fleming I, Fisslthaler B, Hermann C, Busse R, Zeiher AM. Activation of nitric oxide synthase in endothelial cells by Akt-dependent phosphorylation. Nature. 1999;399(6736):601-605.

11. Fulton D, et al. Regulation of endotheliumderived nitric oxide production by the protein kinase Akt. Nature. 1999;399(6736):597-601.

12. Balligand JL, Feron O, Dessy C. eNOS activation by physical forces: from short-term regulation of contraction to chronic remodeling of cardiovascular tissues. Physiol Rev. 2009;89(2):481-534.

13. Tzima E, et al. A mechanosensory complex that mediates the endothelial cell response to fluid shear stress. Nature. 2005;437(7057):426-431.

14. Conway D, Schwartz MA. Lessons from the endothelial junctional mechanosensory complex. F1000 Biol Rep. 2012;4:1.
15. Coon BG, et al. Intramembrane binding of VE-cadherin to VEGFR2 and VEGFR3 assembles the endothelial mechanosensory complex. JCell Biol. 2015;208(7):975-986.

16. Baeyens N, Schwartz MA. Biomechanics of vascular mechanosensation and remodeling. $\mathrm{Mol}$ Biol Cell. 2016;27(1):7-11.

17. Wang S, et al. P2Y2 and Gq/G11 control blood pressure by mediating endothelial mechanotransduction. J Clin Invest. 2015;125(8):3077-3086.

18. Coste B, et al. Piezo proteins are pore-forming subunits of mechanically activated channels. Nature. 2012;483(7388):176-181.

19. Ge J, et al. Architecture of the mammalian mechanosensitive Piezo1 channel. Nature. 2015;527(7576):64-69.

20. Woo SH, et al. Piezo 2 is required for Merkel-cell mechanotransduction. Nature. 2014;509(7502):622-626.

21. Ikeda R, Cha M, Ling J, Jia Z, Coyle D, Gu JG. Merkel cells transduce and encode tactile stimuli to drive $A \beta$-afferent impulses. Cell. 2014;157(3):664-675. 
22. Maksimovic S, et al. Epidermal Merkel cells are mechanosensory cells that tune mammalian touch receptors. Nature. 2014;509(7502):617-621.

23. Ranade SS, et al. Piezo1, a mechanically activated ion channel, is required for vascular development in mice. Proc Natl Acad Sci U S A. 2014;111(28):10347-10352.

24. Li J, et al. Piezo1 integration of vascular architecture with physiological force. Nature. 2014;515(7526):279-282.

25. Cahalan SM, Lukacs V, Ranade SS, Chien S, Bandell M, Patapoutian A. Piezo1 links mechanical forces to red blood cell volume. Elife. 2015;4. doi:10.7554/eLife.07370.

26. Syeda R, et al. Chemical activation of the mechanotransduction channel Piezo1. Elife. 2015;4. doi:10.7554/eLife.07369.

27. Locovei S, Wang J, Dahl G. Activation of pannexin 1 channels by ATP through P2Y receptors and by cytoplasmic calcium. FEBS Lett. 2006;580(1):239-244.

28. Gödecke S, Roderigo C, Rose CR, Rauch BH, Gödecke A, Schrader J. Thrombin-induced ATP release from human umbilical vein endothelial cells. Am J Physiol Cell Physiol. 2012;302(6):C915-C923.

29. Lohman AW, et al. Expression of pannexin isoforms in the systemic murine arterial network. J Vasc Res. 2012;49(5):405-416.

30. Bevan JA, Laher I. Pressure and flow-dependent vascular tone. FASEB J. 1991;5(9):2267-2273.

31. Fleming I. The factor in EDHF: Cytochrome P450 derived lipid mediators and vascular signaling [published online ahead of print March 11, 2016]. Vascul Pharmacol. doi:10.1016/j. vph.2016.03.001.

32. Vanhoutte PM, Zhao Y, Xu A, Leung SW. Thirty years of saying NO: Sources, fate, actions, and misfortunes of the endothelium-derived vasodilator mediator. Circ Res. 2016;119(2):375-396.

33. Yamamoto K, Furuya K, Nakamura M, Kobatake E, Sokabe M, Ando J. Visualization of flow- induced ATP release and triggering of $\mathrm{Ca}^{2+}$ waves at caveolae in vascular endothelial cells. J Cell Sci. 2011;124(Pt 20):3477-3483.

34. Bodin P, Bailey D, Burnstock G. Increased flow-induced ATP release from isolated vascular endothelial cells but not smooth muscle cells. Br J Pharmacol. 1991;103(1):1203-1205.

35. Tzima E. Role of small GTPases in endothelial cytoskeletal dynamics and the shear stress response. Circ Res. 2006;98(2):176-185.

36. Poole K, Herget R, Lapatsina L, Ngo HD, Lewin GR. Tuning Piezo ion channels to detect molecular-scale movements relevant for fine touch. Nat Commun. 2014;5:3520.

37. White CR, Frangos JA. The shear stress of it all: the cell membrane and mechanochemical transduction. Philos Trans R Soc Lond B Biol Sci. 2007;362(1484):1459-1467.

38. Fung YC, Liu SQ. Elementary mechanics of the endothelium of blood vessels. J Biomech Eng. 1993;115(1):1-12.

39. Lewis AH, Grandl J. Mechanical sensitivity of Piezo1 ion channels can be tuned by cellular membrane tension. Elife. 2015;4. doi:10.7554/eLife.12088.

40. Cox CD, et al. Removal of the mechanoprotective influence of the cytoskeleton reveals PIEZO1 is gated by bilayer tension. Nat Commun 2016;7:10366.

41. Lee W, et al. Synergy between Piezo1 and Piezo2 channels confers high-strain mechanosensitivity to articular cartilage. Proc Natl Acad Sci U S A. 2014;111(47):E5114-E5122.

42. Retailleau K, et al. Arterial Myogenic Activation through Smooth Muscle Filamin A. Cell Rep. 2016;14(9):2050-2058.

43. Burnstock G, Ralevic V. Purinergic signaling and blood vessels in health and disease. Pharmacol Rev. 2014;66(1):102-192.

44. John K, Barakat AI. Modulation of ATP/ADP concentration at the endothelial surface by shear stress: effect of flow-induced ATP release. Ann Biomed Eng. 2001;29(9):740-751.
45. Lohman AW, Billaud M, Isakson BE. Mechanisms of ATP release and signalling in the blood vessel wall. Cardiovasc Res. 2012;95(3):269-280.

46. Gruenhagen JA, Yeung ES. Investigation of G protein-initiated, $\mathrm{Ca}^{2+}$-dependent release of ATP from endothelial cells. Biochim Biophys Acta. 2004;1693(2):135-146.

47. Good ME, Begandt D, DeLalio LJ, Keller AS, Billaud M, Isakson BE. Emerging concepts regarding pannexin 1 in the vasculature. Biochem Soc Trans. 2015;43(3):495-501.

48. Cinar E, Zhou S, DeCourcey J, Wang Y, Waugh RE, Wan J. Piezo1 regulates mechanotransductive release of ATP from human RBCs. Proc Natl Acad Sci U S A. 2015;112(38):11783-11788.

49. Miyamoto T, et al. Functional role for Piezo1 in stretch-evoked $\mathrm{Ca}^{2+}$ influx and ATP release in urothelial cell cultures. J Biol Chem. 2014;289(23):16565-16575.

50. Lukacs V, et al. Impaired PIEZO1 function in patients with a novel autosomal recessive congenital lymphatic dysplasia. Nat Commun. 2015;6:8329.

51. Buschmann MH, Dieterich P, Adams NA, Schnittler HJ. Analysis of flow in a cone-and-plate apparatus with respect to spatial and temporal effects on endothelial cells. Biotechnol Bioeng. 2005;89(5):493-502.

52. Korhonen $\mathrm{H}$, et al. Anaphylactic shock depends on endothelial $\mathrm{G}_{\mathrm{q}} / \mathrm{G}_{11}$.J Exp Med. 2009;206(2):411-420.

53. Wirth A, et al. G12-G13-LARG-mediated signaling in vascular smooth muscle is required for salt-induced hypertension. Nat Med. 2008;14(1):64-68.

54. Liu C, et al. Depletion of intracellular $\mathrm{Ca}^{2+}$ stores enhances flow-induced vascular dilatation in rat small mesenteric artery. Br J Pharmacol. 2006;147(5):506-515.

55. Sivaraj KK, Takefuji M, Schmidt I, Adams RH, Offermanns S, Wettschureck N. G13 controls angiogenesis through regulation of VEGFR-2 expression. Dev Cell. 2013;25(4):427-434. 